PROCEEDINGS OF THE

AMERICAN MATHEMATICAL SOCIETY

Volume 130, Number 4, Pages 927-933

S 0002-9939(01)06169-X

Article electronically published on August 28, 2001

\title{
AN APPLICATION OF RAMSEY'S THEOREM TO THE BANACH CONTRACTION PRINCIPLE
}

\author{
JAMES MERRYFIELD, BRUCE ROTHSCHILD, AND JAMES D. STEIN, JR.
}

(Communicated by John R. Stembridge)

\begin{abstract}
One of the most fundamental fixed-point theorems is Banach's Contraction Principle, of which the following conjecture is a generalization.

Generalized Banach Contraction Conjecture (GBCC). Let $T$ be a selfmap of a complete metric space $(X, d)$, and let $0<M<1$. Let $J$ be a positive integer. Assume that for each pair $x, y \in X, \min \left\{d\left(T^{k} x, T^{k} y\right): 1 \leq k \leq J\right\} \leq$ $M d(x, y)$. Then $T$ has a fixed point.

Unlike Banach's original theorem (the case $J=1$ ), the above hypothesis does not compel $T$ to be continuous. In this paper we use Ramsey's Theorem from combinatorics to establish the GBCC for arbitrary $J$ in the case when $T$ is assumed to be continuous, and also derive a result which enables us to prove the GBCC when $J=3$ without the assumption of continuity; it is known that the case $J=3$ includes instances where $T$ is not continuous.
\end{abstract}

\section{INTRODUCTION}

The Banach Contraction Principle is one of the most important and heavilyinvestigated fixed-point theorems. The following conjecture generalizes the one originally studied by Banach.

Generalized Banach Contraction Conjecture (GBCC). Let $T$ be a self-map of a complete metric space $(X, d)$, and let $0<M<1$. Let $J$ be a positive integer. Assume that for each pair $x, y \in X, \min \left\{d\left(T^{k} x, T^{k} y\right): 1 \leq k \leq J\right\} \leq M d(x, y)$. Then $T$ has a fixed point.

Banach's original theorem is simply the case $J=1$, in which $T$ is uniformly continuous. We summarize briefly what is known about the GBCC. If $T$ is uniformly continuous, then the GBCC is true for arbitrary $J$ ([3], Theorem 2). If $J=2$, the GBCC is true without any additional assumption on $T$ (2], Theorem 1), and if $J=3$ and $T$ is continuous, the GBCC is true (2], Theorem 2). It is shown in [2] that the case $J=3$ includes examples where $T$ is discontinuous.

Both [2] and [3] make substantial use of combinatorial arguments. In this paper we show that Ramsey's Theorem can be used to prove the GBCC for arbitrary $J$ under the assumption that $T$ is continuous. We also give a proof of a result which has potential application to the proof of the GBCC for arbitrary $J$ without the

Received by the editors March 3, 2000 and, in revised form, May 10, 2000, July 14, 2000, and September 25, 2000.

2000 Mathematics Subject Classification. Primary 05C55, 47H10.

Key words and phrases. Ramsey's Theorem, Banach Contraction Principle, fixed point.

(C)2001 American Mathematical Society 
assumption of continuity; this result can in fact be used to prove the GBCC for $J=3$.

\section{The GBCC for arbitrary $J$ and Continuous $T$}

Two lemmas will be key in establishing the desired result. The first lemma derives a basic consequence of the GBCC hypothesis.

Lemma 1. Let $(X, d)$ be a metric space, $0<M<1, J$ an integer, and let $T$ be a self-map of $X$ satisfying

$$
\min \left\{d\left(T^{j} x, T^{j} y\right): j=1,2, \ldots, J\right\} \leq M d(x, y) \quad \text { for all } x, y \in X .
$$

Then for every $x$, there exists a bounded subsequence of $\left\{T^{n} x: n=1,2, \ldots\right\}$, denoted $\left\{T^{a(n)} x: n=1,2, \ldots\right\}$, such that $a(n+1)-a(n) \leq J$.

Proof. Let $C=\max \left\{d\left(x, T^{k} x\right): k=1,2, \ldots, J\right\}$. We show that we can construct a sequence of integers $a(n)$ such that $d\left(x, T^{a(n)} x\right)<\frac{C}{1-M}$. Let $a(1)=1$. Since $0<1-M<1$, we clearly have $d(x, T x)<\frac{C}{1-M}$. Proceeding inductively, if $d\left(x, T^{a(n)} x\right)<\frac{C}{1-M}$, by hypothesis we have for some $k \leq J$ that $d\left(T^{k} x, T^{a(n)+k} x\right)<$ $\frac{M C}{1-M}$. So, by the triangle inequality, we have

$$
d\left(x, T^{a(n)+k} x\right) \leq d\left(x, T^{k} x\right)+d\left(T^{k} x, T^{a(n)+k} x\right)<C+\frac{M C}{1-M}=\frac{C}{1-M},
$$

and letting $a(n+1)=a(n)+k$ completes the proof.

Note that the conclusion does not require $X$ to be complete.

Although Ramsey's Theorem is of great importance in combinatorics, it may not be known to many fixed-point theorists. We include a statement of the theorem for completeness. The version we use can be found as [1], Theorem A, p. 19.

Ramsey's Theorem. Let $S$ be an infinite set, $n$ a positive integer. Assume that every subset of $S$ of cardinality $n$ has been given one of a finite number of colors. Then there exists an infinite subset $T$ of $S$ such that $T$ is monochromatic; i.e. every subset of $T$ of cardinality $n$ has the same color.

The idea of referring to the assignment of a finite set of values to the sets of cardinality $n$ as 'coloring the subsets of cardinality $n$ ' is a notation that has come about following Ramsey's untimely death at age 28, but it preserves Ramsey's original idea.

The second lemma derives the needed combinatorial result from Ramsey's Theorem.

Lemma 2. Let $m$ and $n$ be positive integers. Let $G$ be a graph whose vertex set is the disjoint union of countably many blocks, each of size $m$. Assume further that each edge has its two endpoints in distinct blocks and that for any $n$ blocks there is at least one edge having its endpoints in two of those blocks. Then there is an infinite path in $G$, visiting no block more than once.

Proof. Number the blocks as $B_{0}, B_{1}, \ldots$, and number the vertices in each block $B_{i}$ as $v(i, 1), v(i, 2), \ldots, v(i, m)$. Color the pairs of natural numbers with $m^{2}+1$ colors by assigning as the color of the pair $(i, j)$, with $i<j$, either some pair $(p, q)$ such that $v(i, p)$ is adjacent in $G$ to $v(j, q)$ or, if there is no edge between $B_{i}$ and $B_{j}$, we assign a special color: "none". Ramsey's Theorem provides an infinite set $H$ of 
natural numbers, every pair of which has the same color. By the hypothesis of the lemma, that color cannot be "none", so suppose it is $(p, q)$. That is, if $i<j$ are both in $H$, then $v(i, p)$ is adjacent to $v(j, q)$. Writing $h(i)$ for the $i$ th element of $H$, we obtain the desired infinite path as $v(h(1), p), v(h(3), q), v(h(2), p), v(h(5), q)$, $v(h(4), p), v(h(7), q), \ldots$

With the preliminaries established, we are now ready to prove the GBCC for the case in which $T$ is continuous.

Theorem 1. Let $T$ be a continuous self-map of a complete metric space $(X, d)$, and let $0<M<1$. Let $J$ be a positive integer. Assume that for each pair $x, y \in X, \min \left\{d\left(T^{k} x, T^{k} y\right): 1 \leq k \leq J\right\} \leq M d(x, y)$. Then $T$ has a fixed point.

Proof. Let $x$ be an arbitrary point in $X$. Let $\langle i, k\rangle=d\left(T^{i} x, T^{k} x\right)$, and for any real number $r$, let $[r]$ denote the greatest integer less than or equal to $r$.

From Lemma 1, there is a sequence $\left\{n_{i}: i=1,2, \ldots\right\}$ such that $\left\langle 0, n_{i}\right\rangle \leq K$ and $n_{i+1}-n_{i} \leq J$. Applying the GBCC hypothesis to each pair of points $x$ and $T^{n_{i}} x$ results in sequences $\left\{q_{i j}: j=1,2, \ldots\right\}$ (one sequence for each $i$ ) such that $\left\langle q_{i j}, n_{i}+q_{i j}\right\rangle \leq K M^{j}$ and $q_{i j+1}-q_{i j} \leq J$. If we let $q=q_{i j}$, then $j \geq[q / J] \geq$ $q / J-1$, in which case $\left\langle q, n_{i}+q\right\rangle \leq K M^{q / J-1}=K_{0} Q^{q}$, where $K_{0}=K / M$ and $Q=M^{1 / J}<1$.

Note that if we have $\left\langle q, n_{i}+q\right\rangle \leq K_{0} Q^{q}$ and $\left\langle q, n_{j}+q\right\rangle \leq K_{0} Q^{q}$ for two different integers $i$ and $j$, by the triangle inequality we have

$$
\left\langle n_{i}+q, n_{j}+q\right\rangle \leq\left\langle n_{i}+q, q\right\rangle+\left\langle q, n_{j}+q\right\rangle \leq 2 K_{0} Q^{q} .
$$

We now introduce some terminology. We say that an integer $q$ is represented if there are infinitely many integers $i$ for which $\left\langle q, n_{i}+q\right\rangle \leq K_{0} Q^{q}$. If $q$ is represented and $\left\langle q, n_{i}+q\right\rangle \leq K_{0} Q^{q}$, we say that $i$ is a representative of $q$. If $A$ is a set of integers, let $r(A)=\{q: q \in A, q$ is represented $\}$ and $R(A)=\{i: \exists q \in$ $r(A)$ such that $i$ is a representative of $q\}$.

Now let $A$ be a set of $J$ consecutive integers. The requirement that $q_{i j+1}-q_{i j} \leq J$ combined with the pigeonhole principle shows that at least one member of $A$ must be represented. Moreover, for all but finitely many $i, \exists q \in r(A)$ such that $i$ is a representative of $q$; i.e. for some integer $I_{0}, i \geq I_{0} \Rightarrow i \in R(A)$.

Again, let $A$ be a set of $J$ consecutive integers. We claim there is an integer $\lambda(A)$ with the following property: if $m \geq \lambda(A)$, some integer of the form $n_{i}+q$ belongs to the set $\{m, m+1, \ldots, m+2 J-1\}$, where $q \in r(A)$ and $i$ is a representative of $q$. We have seen $\exists I_{0}$ such that $i \geq I_{0} \Rightarrow i \in R(A)$. Let $\lambda(A)=\max \{j: j \in A\}+n_{I_{0}}$. Since each $i$ with $i \geq I_{0}$ is a representative of some $q \in r(A)$, we see that if $i+1$ is a representative of $q^{\prime} \in r(A)$ and if $i$ is a representative of $q \in r(A)$, then $n_{i+1}+q^{\prime}-\left(n_{i}+q\right)=\left(n_{i+1}-n_{i}\right)+\left(q^{\prime}-q\right) \leq 2 J$.

Finally, if $A$ is a set of $J$ consecutive integers, let $N Q(A)=\left\{n_{i}+q: q \in r(A)\right.$ and $i$ is a representative of $q$. We observe that if $A_{1}, \ldots, A_{2 J+1}$ are disjoint sets of $J$ consecutive integers each, then if $a=\max \left\{\lambda\left(A_{k}\right): k=1,2, \ldots, 2 J+1\right\}$ and $m \geq a$, any set $\{m, m+1, \ldots, m+2 J-1\}$ must contain an integer common to two sets $N Q\left(A_{j}\right)$ and $N Q\left(A_{k}\right)$ for which $j \neq k$. By partitioning the sequence $\{a, a+1, a+2, \ldots\}$ into blocks of length $2 J$ and applying the pigeonhole principle, we see that there must be two sets $N Q\left(A_{j}\right)$ and $N Q\left(A_{k}\right)$ with $j \neq k$ which have infinitely many integers in common. Again by the pigeonhole principle, there must be integers $q \in A_{j}$ and $q^{\prime} \in A_{k}$ such that there are infinitely many integers in the 
intersection of $N Q\left(A_{j}\right)$ and $N Q\left(A_{k}\right)$ that can be expressed both in the form $n_{i}+q$ (as an integer in $N Q\left(A_{j}\right)$ ) and $n_{p}+q^{\prime}$ (as an integer in $N Q\left(A_{k}\right)$ ).

We now regard each integer as a vertex in a graph, and partition the integers into a disjoint union of blocks $B_{k}=\{(k-1) J+1,(k-1) J+2, \ldots, k J\}$ for $k=1,2, \ldots$. We say that two vertices $q$ and $q^{\prime}$ in distinct blocks $B_{j}$ and $B_{k}$ respectively are connected by an edge if there are infinitely many integers which can be expressed in both the forms $n_{i}+q$ and $n_{p}+q^{\prime}$. The argument in the preceding paragraph shows that for any collection of $2 J+1$ blocks, at least one edge has endpoints in two distinct blocks.

We now apply Lemma 2 to conclude that there is an infinite path through the graph visiting no block more than once. Denote the vertices in this path in the order traversed by $\left\{r_{j}: j=1,2, \ldots\right\}$. Now choose sequences of integers $\left\{s_{j}: j=1,2, \ldots\right\}$ and $\left\{t_{j}: j=1,2, \ldots\right\}$ from among the $\left\{n_{j}: j=1,2, \ldots\right\}$ with the following three properties:

(1) If $r_{j}$ belongs to block $B_{k}$, both $r_{j}+s_{j}$ and $r_{j}+t_{j}$ belong to $N Q\left(B_{k}\right)$.

(2) $r_{j}+t_{j}=r_{j+1}+s_{j+1}$.

(3) $r_{j}+s_{j}<r_{j+1}+s_{j+1}$.

We now consider the sequence of iterates with exponents $r_{j}+s_{j}$. Note that

$$
\sum_{j=1}^{\infty}\left\langle r_{j}+s_{j}, r_{j+1}+s_{j+1}\right\rangle=\sum_{j=1}^{\infty}\left\langle r_{j}+s_{j}, r_{j}+t_{j}\right\rangle \leq \sum_{j=1}^{\infty} 2 K_{0} Q^{r_{j}} .
$$

Since the $\left\{r_{j}: j=1,2, \ldots\right\}$ are all distinct, the above series converges, and so the sequence of iterates is a Cauchy sequence. We show that this results in a fixed point for $T$. Assume that $T^{m_{i}} x \rightarrow z$. By passing to a subsequence if necessary, we can also assume that $m_{i+1}>m_{i}+J$ for all $i$. Since $T$ is continuous, note that for $1 \leq j \leq J$ we have $T^{m_{i}+j} x \rightarrow T^{j} z$. Define $L_{j}=T^{j} z$ for $0 \leq j \leq J$. We show that $L_{j+1}=L_{j}$ for some $j<J$; since $T\left(L_{j}\right)=L_{j+1}$ this will demonstrate that $L_{j}$ is a fixed point of $T$.

Applying the GBCC hypothesis to the pair $(x, T x)$ enables us to conclude, as in the second paragraph of this proof, that for each integer $i$ we can find an integer $j_{i}$ with $0 \leq j_{i} \leq J-1$ and $d\left(T^{m_{i}+j_{i}} x, T^{m_{i}+j_{i}+1} x\right) \leq M^{r_{i}} d(x, T x)$, where $r_{i} \rightarrow \infty$. By the pigeonhole principle, there is an integer $k$ with $0 \leq k \leq J-1$ and $j_{i}=k$ for infinitely many $i$. For those $i$ for which $j_{i}=k$ for infinitely many $i$ we have

$$
\begin{aligned}
d\left(L_{k}, L_{k+1}\right) & \leq d\left(L_{k}, T^{m_{i}+k} x\right)+d\left(T^{m_{i}+k} x, T^{m_{i}+k+1} x\right)+d\left(T^{m_{i}+k+1} x, L_{k+1}\right) \\
& \leq d\left(L_{k}, T^{m_{i}+k} x\right)+M^{r_{i}} d(x, T x)+d\left(T^{m_{i}+k+1} x, L_{k+1}\right) .
\end{aligned}
$$

Each of these three terms approaches 0 as $i \rightarrow \infty$, completing the proof.

We note that the argument given in Lemmas 1 and 2 and Theorem 1 also establishes the following proposition, which could have applications in environments other than fixed-point theory.

Proposition 1. Let $(X, d)$ be a metric space, let $J$ be a positive integer, and let $0<M<1$. Assume that $\left\{x_{i}: i=1,2, \ldots\right\}$ is a sequence in $X$ such that the following condition holds:

$$
\min \left\{d\left(x_{i+k}, x_{j+k}\right): k=1,2, \ldots, J\right\} \leq M d\left(x_{i}, x_{j}\right) \quad \text { for all integers } i \text { and } j .
$$

Then $\left\{x_{i}: i=1,2, \ldots\right\}$ has a Cauchy sub-sequence. 


\section{Results Pertaining to the GBCC When $T$ MAY Be Discontinuous}

In this section we prove that a self-map $T$ satisfying the GBCC hypothesis which can be shown to have a Cauchy sequence of iterates such that the difference between exponents is bounded has a fixed point. The proof does not require $T$ to be continuous, and may therefore be useful in establishing the GBCC in this case.

Theorem 2. Let $T$ be a self-map of a complete metric space $(X, d)$, and let $0<M<1$. Let $J$ be a positive integer. Assume that for each pair $x, y \in X$, $\min \left\{d\left(T^{k} x, T^{k} y\right): 1 \leq k \leq J\right\} \leq M d(x, y)$. Suppose we have obtained points $x, z \in X$, a sequence $\left\{n_{i}: i=1,2, \ldots\right\}$, and an integer $N$ such that $T^{n_{i}} x \rightarrow$ $z, n_{i+1} \leq n_{i}+N$. Then $T$ has a fixed point.

Proof. We start by constructing a doubly-indexed family $\{n(j, i): j=0,1, \ldots ; i=$ $1,2, \ldots\}$ to be used as powers of $T$ which operate on $x$. Let $n(0, i)=n_{i}$ for $i=1,2, \ldots$. Apply the GBCC hypothesis to all pairs of the form $\left(T^{n(0, i)} x, z\right)$. To each integer $n(0, i)$ there is an integer $j(0, i)$ such that $1 \leq j(0, i) \leq J$ and

$$
d\left(T^{n(0, i)+j(0, i)} x, T^{j(0, i)} z\right) \leq M d\left(T^{n(0, i)} x, z\right) .
$$

If $1 \leq j \leq J$, either there exist infinitely many $i$ for which $j(0, i)=j$, or not. By choosing $I(0)$ sufficiently large, we can ensure that if $i \geq I(0)$ and $j=j(0, i)$, then $j(0, k)=j$ for infinitely many $k$. Combining this with (1) and the fact that $T^{n(0, i)} x \rightarrow z$ enables us to regard $\left\{T^{n(0, i)+j(0, i)} x: i \geq I(0)\right\}$ as the union of finitely many disjoint sequences (in the sense that the powers of $T$ form disjoint sequences), each of which converges to some $T^{j} z$ with $1 \leq j \leq J$.

Let $n(1, i)=n(0, i)+j(0, i)$ for $i \geq I(0)$ and repeat the process, applying the GBCC hypothesis to all pairs of the form $\left(T^{n(1, i)} x, T^{j(0, i)} z\right)$ for $i \geq I(0)$. To each integer $n(1, i)$ there is an integer $j(1, i)$ such that $1 \leq j(1, i) \leq J$ and

$$
d\left(T^{n(1, i)+j(1, i)} x, T^{j(1, i)+j(0, i)} z\right) \leq M d\left(T^{n(1, i)} x, T^{j(0, i)} z\right) .
$$

As before, by choosing $I(1) \geq I(0)$ sufficiently large, we can regard $\left\{T^{n(1, i)+j(1, i)} x\right.$ : $i \geq I(1)\}$ as the union of finitely many disjoint sequences, each of which converges to some $T^{j} z$ with $2 \leq j \leq 2 J$. Here, each of the disjoint sequences obtained in the previous step is, except for finitely many terms, decomposed into the union of disjoint sequences.

Let $n(2, i)=n(1, i)+j(1, i)$ for $i \geq I(1)$ and repeat the process inductively. For each integer $k$, we obtain an integer $I(k)$ with $I(0) \leq I(1) \leq \cdots \leq I(k)$, and sequences $\{n(k, i): i=1,2, \ldots\}$ and $\{j(k, i): i=1,2, \ldots\}$ such that $1 \leq j(k, i) \leq$ $J$ for all $k$ and $i$ such that $i \geq I(k), n(k+1, i)=n(k, i)+j(k, i)$ for $i \geq I(k)$, and we can regard $\left\{T^{n(k, i)+j(k, i)} x: i \geq I(k)\right\}$ as the union of finitely many disjoint sequences, each of which converges to some $T^{j} z$ with $k \leq j \leq k J$.

Note that if $i \geq I(k-1)$, then

$$
\begin{aligned}
n(k, i) & =n(k-1, i)+j(k-1, i)=n(k-2, i)+j(k-2, i)+j(k-1, i) \\
& =\cdots=n(0, i)+j(0, i)+\cdots+j(k-1, i) .
\end{aligned}
$$

Since $1 \leq j(0, i) \leq J, \ldots, 1 \leq j(k-1, i) \leq J$, we see that

$$
n(0, i)+k \leq n(k, i) \leq n(0, i)+k J \quad \text { if } i \geq I(k-1) .
$$


Observe from (31) that, by letting $k=(J+1)^{p}$, if $i \geq I\left((J+1)^{p}-1\right)$, we have (4)

$$
n(0, i)+(J+1)^{p} \leq n\left((J+1)^{p}, i\right) \leq n(0, i)+(J+1)^{p} J<n(0, i)+(J+1)^{p+1} .
$$

Fix $p$, and choose integers $q$ and $r$ with $r>q \geq I\left((J+1)^{p}-1\right)$. Consider the set of integers $F=\left\{n\left((J+1)^{k}, i\right): 0 \leq k \leq p, q \leq i \leq r\right\}$. Note that, from (4),

$$
\begin{aligned}
n(0, q)+1 & \leq n(0, i)+1 \leq n\left((J+1)^{k}, i\right) \\
& \leq n(0, i)+(J+1)^{k+1} \leq n(0, r)+(J+1)^{p+1} .
\end{aligned}
$$

So the integers in $F$ are contained in an interval of length $\leq n(0, r)+(J+1)^{p+1}-$ $(n(0, q)+1)<(r-q) N+(J+1)^{p+1}$, since $n(0, i+1)-n(0, i) \leq N$ for any $i$. The number of expressions of the form $n\left((J+1)^{k}, i\right)$ with $0 \leq k \leq p$ and $q \leq i \leq r$ is $(p+1)(r-q+1)$. We want to ensure that the number of expressions referred to above exceeds the length of the interval in which those expressions are contained. Let $p=N$. If $r-q>(J+1)^{N+1}$ we will have

$$
\begin{aligned}
(p+1)(r-q+1) & =(N+1)(r-q+1)>(N+1)(r-q) \\
& =(r-q) N+(r-q)>(r-q) N+(J+1)^{N+1} .
\end{aligned}
$$

Consequently, if $r-q>(J+1)^{N+1}$, in the interval from $n(0, q)+1$ to $n(0, r)+$ $(J+1)^{N+1}$ we must have integers $k$ and $l$ with $0 \leq k, l \leq N$ and integers $i$ and $j$ with $q \leq i, j \leq r$ and $n\left((J+1)^{k}, i\right)=n\left((J+1)^{l}, j\right)$, with either $k \neq l$ or $i \neq j$ or both. However, since the integers $n(1, i), n(J+1, i), \ldots, n\left((J+1)^{N}, i\right)$ are all distinct, we must have $i \neq j$. But, from (3),

$$
\begin{array}{ll}
n\left((J+1)^{k}, i\right)=n(0, i)+a & \text { with } a \leq(J+1)^{N+1} \\
n\left((J+1)^{l}, j\right)=n(0, j)+b & \text { with } b \leq(J+1)^{N+1}
\end{array}
$$

Since $i \neq j, a \neq b$. Therefore, in the interval from $n(0, q)+1$ to $n(0, r)+(J+1)^{N+1}$ we have found a term belonging to two sequences, one which converges to $T^{a} z$ and one which converges to $T^{b} z$.

Now choose a sequence $i(1)<i(2)<\cdots$ with $i(1)>I\left((J+1)^{N}-1\right)$, and such that both $i(k+1)-i(k)>(J+1)^{N+1}$ and $n(0, i(2 k+1))+1>n(0, i(2 k))+(J+1)^{N+1}$ for $k=1,2, \ldots$ These restrictions ensure that the intervals from $n(0, i(2 k-1))+1$ to $n(0, i(2 k))+(J+1)^{N+1}$ are disjoint, and of sufficient length. Therefore, as in the preceding paragraph, each of the intervals from $n(0, i(2 k-1))+1$ to $n(0, i(2 k))+$ $(J+1)^{N+1}$ contains an integer which is a member of two sequences, one of which converges to $T^{a} z$ and one which converges to $T^{b} z$ for some pair of integers $a$ and $b$ with $a \neq b$ and both $a$ and $b$ less than or equal to $(J+1)^{N+1}$. We say that the pair $(a, b)$ is associated with that interval.

By the pigeonhole principle, there is some pair $(r, s)$ of integers with $r \neq s$ and $1 \leq r, s \leq(J+1)^{N+1}$ that is associated with infinitely many disjoint intervals, and so we can find a sequence of the form $\left\{T^{m_{i}} x: i=1,2, \ldots\right\}$ such that each integer $m_{i}$ has the form $n\left(0, r_{k}\right)+r=n\left(0, s_{k}\right)+s$. This sequence converges to both $T^{r} z$ and $T^{s} z$. So $T^{r} z=T^{s} z$, and by [2], Lemma 1, the proof is complete.

Corollary. If $T$ satisfies the $G B C C$ condition for $J=3$, then $T$ has a fixed point.

Proof. In [2], Theorem 2, it is shown that the hypotheses of the above theorem are satisfied with $N=2$. 


\section{ACKNowledgement}

The authors would like to express their appreciation to the referee for suggestions which significantly clarified the exposition in this paper.

\section{REFERENCES}

1. Ronald Graham, Bruce Rothschild, and Joel Spencer, Ramsey Theory, Wiley-Interscience, New York, 1980. MR 82b:05001

2. Jacek R. Jachymski, Bernd Schroder, and James D. Stein, Jr., A connection between fixedpoint theorems and tiling problems, J. Combin. Theory Ser. A 87 (1999), 273-286. MR 2000g:54075

3. Jacek R. Jachymski and James D. Stein, Jr., A minimum condition and some related fixedpoint theorems, J. Austral Math. Soc. Ser. A 66 (1999), 224-243. MR 2000a:54072

Long Beach Polytechnic High School, 1600 Atlantic Ave., Long Beach, California 90813

E-mail address: kmerry@csulb.edu

Current address: Department of Mathematics, University of California at Berkeley, Berkeley, California 94720

Department of Mathematics, University of California at los Angeles, 405 Hilgard Ave., Los Angeles, California 90024

E-mail address: blr@math.ucla.edu

Department of Mathematics, California State University at Long Beach, 1250 BellFlower Blvd., Long Beach, California 90840

E-mail address: jimstein@csulb.edu 